\title{
METILFENIDATO Y MODAFINILO: JUSTICIA IGUALITARIA Y POLÍTICAS DE ACCESO AL MEJORAMIENTO COGNITIVO FAR- MACOLÓGICO
}

\author{
Daniel Loewe ${ }^{1}$
}

Resumen: El artículo investiga criterios de acceso a tecnologías de mejoramiento cognitivo farmacológico, especialmente modafinilo y metilfenidato, en caso de adultos sanos. Desde la perspectiva de la justicia igualitaria, tomando como referencia la teoría de justicia de Rawls, se argumenta a favor de una política de acceso libre mediante mecanismos de mercado, pero facilitado en caso de los peor situados de la sociedad.

Palabras clave: justicia igualitaria, igualdad de oportunidades, mejoramiento cognitivo farmacológico, metilfenidato, modafinilo

Methylphenidate and Modafinil: egalitarian justice and policies of access to pharmacological cognitive enhancement

Abstract: The article addresses criteria for access to pharmacological cognitive enhancement technologies, especially modafinil and methylphenidate, to healthy adults. From the perspective of equalitarian justice and taking as point of reference Rawls theory of justice it argues in favor of a policy of open market access, but facilitated for the worst-off.

Key words: egalitarian justice, equality of opportunities, cognitive pharmacological enhancement, methylphenidate, modafinil

Metilfenidato e Modafinil: justiça igualitária e políticas de acesso a melhoria cognitiva farmacológica

Resumo: o artigo investiga os critérios para o acesso às tecnologias de aprimoramento cognitivo farmacológica, especialmente o modafinil e o metilfenidato, no caso de adultos saudáveis. Na perspectiva da justiça igualitária, tendo como referência a teoria da justiça de Rawls, argumenta-se a favor de uma política de acesso livre através de mecanismos de mercado, porém com acesso facilitado no caso dos piores situados na sociedade.

Palavras-chave: justiça igualitária, igualdade de oportunidades, aprimoramento cognitivo farmacológico, metilfenidato, modafinil

\footnotetext{
${ }^{1}$ Escuela de Gobierno, Universidad Adolfo Ibáñez, Chile Correspondencia: daniel.loewe@uai.cl
} 
En la actualidad se llevan a cabo amplias discusiones académicas en torno al mejoramiento cognitivo farmacológico (en adelante: $\mathrm{MCF})^{2}(1-3)$. Además de teóricas, ellas tienen un cierto grado de urgencia. Por una parte, hay fármacos, como metilfenidato y modafinilo, que reclaman el mejoramiento de facultades cognitivas (como memoria, enfoque, resolución de problemas, incremento energético, disminución de la necesidad de descanso) en personas sanas. Si bien la extensión del mejoramiento está en disputa, y hay evidencia de que el mejoramiento de algunos tipos de cognición menoscaba otros(4), abunda investigación en substancias que ofrecerán nuevas oportunidades de mejoramiento en las próximas décadas(5). Por otra parte, la investigación empírica indica un uso amplio de MCF por parte de académicos y universitarios. Un foco de las discusiones refiere a la regulación del acceso.

En este artículo discuto, desde la perspectiva de la justicia igualitaria, criterios de acceso a tecnologías de MCF, específicamente metilfenidato y modafinilo, para adultos sanos. En la argumentación asumo que el MCF es seguro. Procedo en siete pasos. Primero presento evidencia empírica acerca del uso de metilfenidato y modafinilo entre estudiantes en Chile y Latinoamérica. En las secciones 2 y 3 examino las implicancias de la teoría rawlsiana en el caso del MCF. En la 4 refiero las dificultades de la distinción entre restauración y mejoramiento. Luego presento seis criterios para regular el acceso basado en consideraciones de justicia igualitaria. En la sección 6 examino críticas al criterio defendido. Finalmente, cualifico el resultado en razón del riesgo a la salud.

\section{Uso de metilfenidato y modafinilo entre estu- diantes}

En Latinoamérica hay pocos estudios estadísticos amplios sobre el uso no médico de psicotrópicos cognitivos. Pero aunque la información es limitada, indica un uso bastante común comparable con la evidencia internacional(6,7). Entre 1997

\footnotetext{
${ }^{2}$ Como definición de trabajo y de un modo corriente entiendo como "mejoramiento cognitivo" (cognitive enhancement) intervenciones que aspiran a mejorar el funcionamiento mental (memoria, foco, resolución de problemas, incremento de la energía, disminución de la necesidad de descanso, etc.), más allá de lo que es necesario para mantener y restaurar la salud(13). En sección 4 discuto este tipo de definiciones.
}

y 2008 no habría publicaciones SciELO sobre el uso de metilfenidato en Brasil(8). La mayoría de las investigaciones se focalizan en el consumo de drogas lícitas e ilícitas por parte de estudiantes, sin preguntar por modafinilo y metilfenidato $(9,10)$. Los pocos estudios disponibles se concentran en estudiantes del sector salud. Según una revisión de cuatro bases de datos entre 1990 y 2012, con artículos en inglés, portugués y español sobre el uso de metilfenidato entre estudiantes de medicina, la prevalencia alcanzaría el 16\% sin distinción entre género(11).

Es usual relacionar el MCF con situaciones académicas demandantes y el estrés asociado. El estrés depende de diversos factores (biológicos, psicológicos, educativos, capacidades cognitivas, las propias aspiraciones académicas). La cualidad de la presión experimentada depende de factores como salud, ánimo, situación económica y familiar, motivación, relaciones personales y religiosidad. Pero siguiendo la relación entre altos niveles de estrés y la tendencia al MCF, los estudiantes del sector de salud debiesen estar entre los usuarios más comunes $^{3}$. Además, este sector tiene características propias (se conocen las consecuencias del uso y es más fácil el acceso), lo que torna implausible generalizar esta prevalencia ${ }^{4}$.

Según un estudio con 122 estudiantes de medicina en la Universidad Nacional de Buenos Aires(12), el $44 \%$ consume substancias para estudiar más tiempo: $72,5 \%$ café, $58,8 \%$ bebidas energizantes, y $45 \%$ substancias psicotrópicas, principalmente modafinilo (31,7\%) y metilfenidato $(13,72 \%)$. Según un estudio en diversas facultades de la Universidad de Manizales en Colombia sobre consumo de anfetaminas y substancias relacionadas(15), con un pool representativo de 309 estudiantes sobre una población de 3616, el $12,1 \%$ las utiliza por razones académicas, sobre todo metilfenidato $(38,6 \%) .71 \%$ de los usuarios reportó resultados positivos.

La afirmación común en Chile es que el consumo de substancias psicotrópicas aumenta, pero no ha-

${ }^{3}$ Si bien medicina lidera los estudios más exigentes, enfermería se percibe como más exigente(14).

${ }^{4}$ Sin embargo, mis - no representativas - conversaciones con estudiantes universitarios de otras disciplinas indican un uso bastante común de modafinilo y metilfenidato, sobre todo en periodos de examen. 
bría alcanzado niveles preocupante(16). Hay algunos estudios sobre el uso de modafinilo por parte de estudiantes del sector salud. Según un estudio estadísticamente no representativo en estudiantes de medicina de la Universidad de Chile(17), 16 de 121 declararon usar modafinilo o pseudoephedrina. Pero el uso efectivo podría ser mayor: en otro estudio en la Universidad Austral de Chile(18) con estudiantes de medicina y enfermería, el 37\% reportó consumo de modafinilo en algún momento de sus estudios. En otro estudio en la misma Universidad en estudiantes de medicina, el 49\% reportó consumo de substancias psicotrópicas al menos una vez durante sus estudios(19): el $77.6 \%$ de ellos usó estimulantes del sistema nervioso central (68\% de ellos sin receta) como modafinilo $(68,5 \%)$, que corresponde al mayor consumo.

\section{Igualitarismo rawlsiano}

Una teoría de justicia igualitaria muy influyente es el "igualitarismo de la suerte" (20-22). Ella da una respuesta a por qué la (des)igualdad material (definida según algún conjunto de bienes) es relevante, basándose en una intuición sobre el igual valor de los individuos como agentes morales, y así en una distinción entre suerte y agencia (o entre circunstancias y decisiones): debido a la igualdad moral se asume como punto de partida igualdad distributiva, y debido al valor moral de la agencia se considera que las desviaciones distributivas solo son legítimas si puede retrotraerse a la agencia, mientras que las debidas a la suerte (no asumida mediante agencia) son ilegítimas:

"(T)here is injustice in the distribution when the inequality of goods reflects not such things as differences in the arduousness of different people's labors, or people's different preferences and choices with respect to income and leisure, but myriad forms of lucky und unlucky circumstance" (23).

Posiciones igualitarias "sociales", "relacionales" o "democráticas" (24-27) articulan otras respuestas a por qué la (des)igualdad es importante. Ellas juzgan la justicia desde la calidad de las relaciones sociopolíticas consideradas relevantes. Si bien la de Rawls es una teoría de "igualdad democrática", también en ella la distinción entre agencia y suerte es fundamental. Pero se trata de un ideal político basado en una idea fundamental de democracia: la justicia aspira a neutralizar los efectos de circunstancias inmerecidas en la vida, porque la regulación de las desigualdades se sigue de un principio de reciprocidad que gobierna las relaciones entre los miembros de la sociedad 5 . Desigualdades distributivas basadas en elección son legítimas, pero desigualdades no merecidas (debidas a la suerte) deben ser neutralizadas". Los "accidentes" y "contingencias" no merecidos refieren a la lotería social y natural: el estatus social y económico inicial así como las fortalezas cognitivas y naturales genética y contextualmente dadas afectan las posibilidades en la vida, pero son "arbitrary from a moral point of view" (28:72). Rawls estipula que el objeto de la justicia son las instituciones sociales más importantes. Lo justo o injusto es "the way the basic structure of society makes use of the natural differences and permits them to affect the social fortune of citizens, their opportunities in life, and the actual terms of cooperation between them" (29:337). Se trata de una "consideración socioestructural de la justicia": los activos naturales no deben ser directamente modificados, sino las instituciones políticas y sociales que los transforman en (des)ventajas.

Para esto Rawls propone el Principio de la Igualdad Justa de Oportunidades, que exige que individuos de igual talento y motivación tengan igual posibilidad de alcanzar puestos y posiciones sociales(28:73). Pero ya que talento y motivación están genética y socialmente basados, se requiere un principio compensatorio para quienes sufren desventajas relacionadas con circunstancias no escogidas: el Principio de la Diferencia asegura que las desigualdades sociales y económicas maximicen las perspectivas de vida de los peor

\footnotetext{
5 Según Rawls, la sociedad es un sistema de cooperación y los principios de justicia gobiernan la distribución de sus beneficios y cargas $(27,28)$. Esos principios son aquellos que individuos libres e iguales pueden aceptar razonable y mutuamente. Esta concepción implica igualdad distributiva de un cierto conjunto de bienes (bienes primarios), porque es parte de los principios que cualquiera tiene buenas razones para aceptar. Rawls afirma que "democratic equality properly understood requires something like the difference principle" (27:49;28:65-66). En el artículo recurro a la teoría de Rawls. Pero lo que sostengo es también aplicable al igualitarismo de la suerte.

${ }^{6}$ Hay muchos modos de entender el contraste entre suerte y agencia, y así muchos modos de trazar la frontera entre ellos(20-22,30). No consideraré estas discusiones. Para mi argumento basta con que haya una diferencia moralmente relevante entre ellos, y que la suerte incluya la lotería social y natural.
} 
situados(27:42-43). Ambos aspiran a mitigar los efectos de circunstancias no merecidas mediante instituciones sociales y políticas, y no mediante intervención en dotaciones naturales.

Rawls asume así la relevancia moral de la diferencia entre (i) personas en tanto sujetos a los que se distribuyen bienes, y (ii) los bienes que se distribuyen a personas(31:126). La justicia aspira a distribuir bienes exteriores a los individuos, y estos son considerados como dados y no modificables. ¿Es esta diferencia sostenible desde la perspectiva de la justicia igualitaria?

\section{Intervenciones genéticas y $\mathrm{MCF}$}

Activos naturales (talentos, fortalezas, etc.) y oportunidades sociales actúan juntas. Así, una capacidad natural puede ser ventajosa o no según la estructura social (considere, por ej. dislexia en una sociedad agraria preindustrial). Y en toda sociedad moderna que premia el esfuerzo hay habilidades naturales cuyo nivel de presencia o ausencia se correlaciona con probabilidades crecientes de éxito o fracaso ${ }^{7}$. Por ejemplo, hay enfermedades que al debilitar la mente y el cuerpo afectan esas habilidades e impiden que los individuos sean competidores normales por ventajas (Chorea Huntington, Tay-Sach, Alzheimer, fibrosis cística, etc.). Si fuese posible evitar estas enfermedades, por ejemplo mediante intervención genética, y así mejorar directamente la mala suerte de la lotería natural, no sería persuasivo argüir que debiésemos limitarnos a compensar con bienes materiales a los que las padecen. Para el igualitarismo es atractivo el argumento a favor de la intervención genética para tratar o impedir enfermedades serias.

El modelo limitado de funcionamiento normal de igualdad de oportunidades de Buchanan recoge este aspecto y así va más allá de la consideración socioestructural de la justicia. La distinción entre terapia y mejoramiento es central: "terapia" serían intervenciones que aspiran a prevenir o curar o reducir los efectos de una enfermedad, entendida como una desviación adversa de un funcionamiento humano típico de la especie, mientras que "mejoramiento" aspiraría a afectar factores (talento, inteligencia,

\footnotetext{
${ }^{7}$ Hay evidencia empírica de correlación entre altos niveles de talento, de educación, de salario y de otras ventajas, como esperanza de vida, salud, etc.(33-37).
}

fortaleza, etc.) aunque no estén relacionados con enfermedad. El objetivo de la medicina sería mantener a los individuos cerca del funcionamiento normal. Enfermedad sería moralmente relevante porque "limits opportunity on the most serious cases, at least by preventing persons from developing the threshold of abilities necessary for being a "normal competitor" in social cooperation" (32:74). Sin embargo, esta concepción es implausible.

Considere el muy discutido caso de un niño con deficiencia de la hormona de crecimiento a causa de un tumor, que como adulto alcanzará 160 $\mathrm{cm}$, y uno con secreción normal de la hormona que como adulto alcanzará la misma altura por su herencia genética. De acuerdo al modelo de Buchanan sería mandatorio el tratamiento hormonal para el primero en razón de la causa de su crecimiento limitado (enfermedad), pero no para el segundo por ser mejoramiento. Pero como vimos, enfermedad es moralmente relevante porque limita la oportunidad. En este sentido, ambos se encuentran en la misma situación: por razones que sobrepasan sus decisiones requieren la hormona de crecimiento para alcanzar un umbral de funcionamiento humano. Tratar diferente casos idénticos en el sentido moral relevante es contraintuitivo y arbitrario(38).

Interpretativamente, la teoría de justicia de Rawls permite superar el modelo de Buchanan, incluyendo formas de mejoramiento ${ }^{8}$ : en la posición original, cada cual tendría un interés en el mejoramiento de sus activos naturales, en razón del interés superior en la realización de los propios planes de vida. No sabemos quiénes somos fuera de la posición original, pero quienquiera que seamos, cada cual estaría interesado en tener las propiedades que faciliten su plan de vida. En la posición original no tenemos un interés particular en llegar a ser un individuo en particular (por ejemplo, aquel que somos) al cual se distribuyen bienes, sino que en llegar a ser un individuo con aquellas propiedades que aumenten la probabilidad de realizar el propio plan de vida, cualquiera sea(39). Si es posible reducir la probabilidad de ser

\footnotetext{
${ }^{8} \mathrm{Al}$ referirse al interés de los individuos en la posición original en tener activos naturales mejores — dados por dotación genética- para así alcanzar mejor sus objetivos, Rawls afirma que "over time a society is to take steps at least to preserve the general level of natural abilities and to prevent the diffusion of serious defects" (28:92). Esto lleva a políticas que aspiren a mejorar la dotación genética.
} 
menoscabado en relación al logro del plan de vida debido a una dotación genética insuficientemente rica, en la posición original optaríamos por políticas que aspiren a mejorar la dotación genética más allá de cualquier umbral general de habilidades humanas ${ }^{9}$. Y este interés debería pesar más que el interés de los padres potenciales en tener la libertad de elegir un descendiente como resultado de la lotería natural. Asumiendo que el crecimiento reducido puede ser un impedimento relevante (si implica una disminución importante de oportunidad), ambos tienen la misma demanda legítima.

Este argumento se extiende al MCF. La recompensa al esfuerzo y el premio a ciertas habilidades cognitivas operativas en sociedades con mecanismos de mercado, implica una relación entre mejoramiento cognitivo y la oportunidad de lograr. En la posición original cada cual estaría interesado en tener acceso a las tecnologías de MCF, para así desarrollar propiedades que tornen más probable la realización del propio plan de vida. Sin embargo, este caso es distinto al del mejoramiento genético, porque cada decisión singular sobre su utilización debe descansar en la voluntad del usuario. Esto corresponde a la prioridad lexicográfica rawlsiana del Principio de la Libertad Igual por sobre el de la Igualdad Justa de Oportunidades: incluso si el MCF incrementase la oportunidad de lograr, cada cual debe disponer de la libertad para decidir sobre su uso. Individuos tienen concepciones del bien y planes de vida diferentes. Tal como un individuo puede decidir no usar sus mejores capacidades cognitivas (por ejemplo, mediante subordinación a una autoridad religiosa), otro debe poder decidir no utilizar las tecnologías de MCF en razón de sus preferencias o concepción del bien. Si bien el igualitarismo nos da buenas razones de principio para no limitar el acceso ${ }^{10}$, la prioridad de la libertad implica que cada cual debe decidir sobre su uso.

\footnotetext{
${ }^{9}$ El argumento depende de la - debatible- existencia de capacidades humanas individuales, cuyo mejoramiento sea valioso para la realización de un plan de vida autónomo. Así, deben ser rechazados todos aquellos mejoramientos que menoscaben la capacidad autonómica.

${ }^{10}$ Hay buenas razones también en el caso de estrategias de mejoramiento no farmacológicas, como nutrición, ejercicio físico, dormir, meditación, estrategias de mnemotécnica, etc. Hay investigación que sugiere que ellas son más eficientes que el mejoramiento farmacológico(40).
}

\section{Terapia y mejoramiento}

En el centro de los debates yace la distinción entre mejoramiento y terapia. La distinción puede realizarse en forma diferente, pero siempre expresa una demanda normativa. Así, encontramos dos temas asociados: el conceptual y el normativo. Corrientemente se acepta que las tecnologías terapéuticas son medios para restaurar capacidades humanas dañadas hasta alcanzar un nivel normal, mientras que las de mejoramiento son medios para llevarlas más allá de este nivel. La primera aspira a hacer posible un funcionamiento humano normal, mientras que la segunda uno mejor que normal. La demanda normativa usual es que la aspiración restaurativa es moral y políticamente valiosa, pero no la de mejoramiento. Por ejemplo, si el usuario de metilfenidato o modafinilo sufre de trastorno con déficit de atención con hiperactividad (ADHD) o de narcolepsia, se considera la aspiración restaurativa y aceptable. Pero si se utilizan sin los síndromes para mejorar habilidades cognitivas, se lo considera mejoramiento y por tanto cuestionable.

Sin embargo, este tipo de distinción conceptual y la demanda normativa asociada son cuestionables. Cualquier recurso a estándares de normalidad es debatible. Primero: ellos están siempre histórica, social y tecnológicamente contenidos. El escándalo por una esperanza estadística de vida de 50 años descansa en las tecnologías disponibles que hacen posible una mayor. Así, es razonable afirmar que la distinción no es significativa(41). Una segunda dificultad surge con la estipulación de las capacidades o habilidades consideradas propiamente humanas, así como con la determinación de los criterios de necesidad y suficiencia. Pero aunque se delimitase una clase de capacidades, se presenta un tercer problema: la estipulación del nivel de normalidad. ¿Es el promedio, o el mejor $20 \%$ o $60 \%$, u otro nivel arbitrario? ${ }^{11}$.

La distinción suele descansar en premisas cuestionables y normativamente cargadas (usualmente naturalistas) sobre qué es apropiado o normal para un ser humano. Una estrategia usual es relacionar la distinción entre restauración y mejoramiento

\footnotetext{
${ }^{11}$ El único caso que evitaría el problema de la arbitrariedad en el establecimiento del nivel de normalidad sería la creación de capacidades completamente nuevas.
} 
con una entre enfermedad y salud. Pero debido a la dificultad para proveer una definición exacta de "enfermedad" y "salud" (42), esta estrategia reproduce problemas similares. Por ejemplo, en su influyente definición, Boorse $(33,43,44)$ relaciona "salud" con funcionamiento normal estadístico de una especie. Como vimos, hay distinciones similares en los debates éticos $(31,32,45,46)$. Sin embargo, la reclamada no-normatividad de esta distinción conceptual descansa en decisiones normativas sobre cuáles son los funcionamientos humanos normales(47).

Sin embargo, distinguir entre enfermedad y salud puede ser productivo en un sentido limitado. Primero: a un nivel intuitivo todos tienen alguna idea, aunque inexacta, sobre esta distinción. Esto es productivo para operacionalizar las teorías, aunque sea aproximativamente. Segundo: aunque diste de claridad y exactitud, la distinción es central en preguntas relativas a la asignación de recursos médicos escasos(48). Este es también el caso del modelo de Buchanan: la diferencia es importante porque en tanto requerimiento de compensación el tratamiento de enfermedades sería más importante que el mejoramiento. Es una distinción normativa débil, según la cual la primera pero no la segunda debiese ser obligatoria.

\section{Criterios de asignación de MCF}

El uso extendido de metilfenidato y modafinilo contrasta con su acceso legal restrictivo ${ }^{12}$. En Chile metilfenidato y heroína pertenecen a la misma categoría(49). El acceso a metilfenidato y modafinilo es mediante receta retenida y receta médica simple respectivamente. Pero hay un gran mercado negro en internet y en las universidades. Siguiendo alguna interpretación de la distinción discutida, se suele aceptar su uso cuando la aspiración es restaurativa. Sin embargo, como discutí (secciones 2 y 3 ), hay buenos argumentos igualitarios para considerar otros criterios de asignación. $\mathrm{Si}$ "there is no reason that neurocognitive enhancement could not help to equalize opportunity in our society" (50:423), disponemos de razones para proponer criterios de asignación que sobrepasen las

\footnotetext{
${ }^{12}$ La posición académica predominante sobre el uso de MCF por parte de estudiantes es crítica $(51,8,12,17,18)$.
}

aspiraciones restaurativas. Discutiré 6 criterios $^{13}$.

Una política de acceso abierto mediante mecanismos de mercado (laissez faire) reflejaría el interés de cada cual en la posición original. Sin embargo, esta opción es objeto de una crítica pertinente: los mejor situados en la sociedad llegarían a estar incluso mejor. Ya que tienen más medios económicos para acceder al MCF, y ya que habilidades cognitivas mejoradas incrementan la capacidad para lograr, esta política se opone a la aspiración igualitaria.

Una política puede evitar ese riesgo al limitar el acceso de mercado al MCF a los cognitivamente menos aventajados. Ella se funda en el interés de cada cual en la posición original en estar en la mejor posición posible en caso de pertenecer a los más desfavorecidos por la lotería natural. Sin embargo, asumiendo que contexto social y habilidades cognitivas se refuerzan mutuamente, y que correspondientemente los cognitivamente más desaventajados pertenecen sobreproporcionalmente a la clase de los peor situados socialmente, algunos de ellos no tendrían acceso si las barreras económicas fuesen significativas.

Sería razonable, por tanto, una política que facilite el acceso a los cognitivamente menos favorecidos. La lógica es consistente con la distinción entre tratamiento y mejoramiento: individuos por debajo de algún umbral cognitivo deberían tener acceso libre y económicamente facilitado al MCF como tratamiento. El punto a discutir sería la estipulación del umbral.

Pero si el fundamento normativo es igualitario, no hay razón para restringir el acceso a los cognitivamente menos favorecidos. De la aspiración de neutralizar los efectos de las circunstancias no merecidas se sigue que debiésemos mejorar la capacidad de cada desaventajado, ya sea por la lotería natural o social. Si el MCF puede mejorar algunas habilidades cognitivas, entonces un principio que las restringe y facilita económicamente a los peor situados por la lotería natural o social, ayudaría a disminuir la brecha entre la oportunidad para

\footnotetext{
${ }^{13}$ Una discusión acerca de las opciones de políticas de acceso en el caso de anfetaminas, metilfenidato y otros mejoradores cognitivos farmacológicos en Dubljević(52,53).
} 
lograr de los menos y de los más aventajados ${ }^{14}$.

Una política extrema facilitaría económicamente el acceso a todos, por ejemplo, subvencionando el producto. Ello tiene cierto sentido: en la posición original cada cual estaría interesado en tener acceso al MCF para mejorar capacidades incrementando la probabilidad de realizar el plan de vida, y esta política facilitaría económicamente el acceso de todos al MCF. Sin embargo, (i) esta política no considera restricciones presupuestarias, siendo inapropiada para contextos de recursos limitados, y (ii) no toma en serio la aspiración igualitaria de mejorar la oportunidad de lograr de los peor situados.

Desde una perspectiva liberal igualitaria sería más justo implementar una política de acceso libre al MCF con facilitación económica para los peor situados como forma de compensación ${ }^{15}$. El acceso facilitado quiebra la espiral de mejoramiento de los mejor situados, ayudando a cerrar la brecha en oportunidad de lograr, y toma en serio las restricciones presupuestarias. A continuación consideraré algunas críticas a esta política.

\section{Críticas}

Primero: no habría diferencia entre (f) y la prohibición del MCF: si los mejor situados disponen de medios económicos para acceder al MCF, la distancia hacia los peor situados se mantendría (i) aunque se facilitase su acceso, o (ii) se prohibiese el acceso a todos. Comparativamente, (f) no implicaría ninguna variación.

Segundo: al ser bienes posicionales, el valor de las capacidades cognitivas se relaciona con que su posesión es desigual. Los talentosos son admirados porque no todos tienen su nivel o tipo de talento. Si mediante MCF cualquiera puede escribir $E l$ Quijote, el talento de Cervantes no sería asunto de admiración.

Tercero: en una economía de mercado las capacidades cognitivas son ventajas comparativas. Si todos las mejoran mediante el MCF, obtendría-

\footnotetext{
${ }^{14}$ Los componentes sociales de la "ambición" que igualitaristas de la suerte consideran parte de una teoría igualitaria(30) están fuera del alcance de esta política.

15 Para un argumento a favor de un mejoramiento moderado basado en compensación: Gesang(54).
}

mos una "carrera armamentista" cognitiva, pero sería una pérdida de tiempo, esfuerzo y dinero(55:328): las ventajas comparativas se mantendrían idénticas (o disminuirían, si el MCF lleva a todos al mismo nivel). Si bien (f) eleva el promedio de capacidades cognitivas en la sociedad, no habría necesariamente un mejor retorno para nadie - pero sí habría uno peor para los que no las usen-.

El primer argumento supone que la posición de los mejor y peor situados para acceder a MCF es idéntica. Pero ese no es el caso: los peor situados disfrutan de acceso garantizado, mientras que los mejor situados deben realizar un trade-off entre sus fines. Presupuestos son limitados, incluso para los mejor situados. Así, es razonable suponer que (f) mejora la oportunidad para lograr de los peor más que la de los mejor situados. Además, hay evidencia que la efectividad del mejoramiento cognitivo es mayor en individuos con menor rendimiento(56-58).

Aunque todos accedan al MCF, sus efectos serían más pronunciados en los cognitivamente menos aventajados. Así (f) promueve la igualdad de oportunidades.

Si la gente accede al MCF, el promedio de habilidad cognitiva aumentará. Pero esto no implica que cada cual vaya a alcanzar el mismo o un nivel similar de habilidades. El MCF no es una píldora mágica que lleve a todos al mismo nivel de habilidad o que incremente en todos la habilidad con la misma proporción. El MCF es efectivo sobre un sustrato natural. Si es así, el promedio aumentará, pero permanecerá alguna, probablemente todavía fuerte, dispersión de capacidades cognitivas. Así, (i) los talentosos seguirán siendo admirados; y (ii) las ventajas comparativas no desaparecerán. Además, si la curva de efectividad del MCF es más pronunciada mientras menos habilidades cognitivas(56-58), el retorno del mercado privilegiará especialmente a los cognitivamente menos aventajados que lo utilicen.

Habilidades cognitivas tienen un valor de retorno en el mercado. Pero su valor no se reduce a este retorno. Ellas no son sólo bienes posicionales(59): aunque los que recurren al MCF no obtuviesen un mejor retorno, tendrían acceso a más oportu- 
nidades para el enriquecimiento de su vida, lo que puede ser productivo para el desarrollo, revisión y consecución de un plan de vida.

La idea de que el incremento del promedio de habilidades cognitivas no implica un mejor retorno de mercado se funda en las suposiciones que (i) el rendimiento global del mercado es fijo y (ii) las interacciones de los individuos son un juego de suma cero. Pero (i) y (ii) son falsas: una economía moderna con más habilidades cognitivas dispone de una mejor oportunidad para crecer y así mejorar el retorno absoluto individual. Además, habilidades cognitivas tienen externalidades sociales positivas no sólo económicas. Por ejemplo, mayor capacidad de resolución de problemas sociales, de desarrollo de nuevas tecnologías y fármacos, etc.

\section{Conclusiones}

Más allá de lo que tematizan los defensores del igualitarismo, sus demandas de justicia para neutralizar las desventajas de la suerte se deben extender (i) a no limitar la libertad para acceder al MCF y (ii) a garantizar un acceso económicamente facilitado a los peor situados. Según Rawls: "We might conjecture that on the long run, if there is an upper bound on ability, we would eventually reach a society with the greatest equal liberty the members of which enjoy the greatest equal talent" (28:92-3). Al promover el talento, (f) promueve la libertad de los miembros de la sociedad.

Sin embargo, este resultado debe ser cualificado ${ }^{16}$. Para el argumento he asumido que el MCF es

\footnotetext{
${ }^{16}$ En otro lugar, examino otras críticas: implicaría engaño y coerción ilegítima de los que no quieren utilizarlas. A mi juicio, ellas no son conclusivas (60).
}

seguro, pero ¿lo es? Hay evidencia de que el uso de metilfenidato indica un riesgo de dependencia moderado. Y si bien modafinilo parece seguro, no disponemos de estudios concluyentes de largo plazo.

Por una parte, la incertidumbre sobre las consecuencias del MCF en la salud debe ser considerada. Si no hay daño en su uso, no hay razones para restringir el acceso. Como regla general: mientras menos seguro sea, o mientras más inciertas sean sus consecuencias en la salud, más se recomienda controlar y restringir el acceso. Esta regla no se basa en la protección de los usuarios voluntarios, sino en la de aquellos que no quieren usarlo por el riesgo de daño, pero que lo utilizan debido a la presión de competencia.

Por otra parte, considerar la incertidumbre no implica que todo daño a la salud o riesgo de ocurrencia veten (f). Se debe sopesar los riesgos contra la aspiración igualitaria de la política y contra la libertad de las personas. De modo general, mientras menos igualitaria sea la sociedad y, correspondientemente, mayor sea la brecha entre la oportunidad de lograr de los peor y los mejor situados, más relevante es la aspiración igualitaria. Si el MCF no es inseguro, o el riesgo de daño moderado es bajo, hay buenas razones igualitarias para apoyar (f) y avanzar así hacia la igualdad de oportunidades.

\section{Agradecimientos}

El artículo se inscribe en el proyecto Fondecyt (1120736).

\section{Referencias}

1. Merkel R, Boer G., Fegert J, et al. Intervening in the Brain. Changing Psyche and Society. Berlin, Heidelberg: Springer; 2007.

2. Schöne-Seifert B, Talbot D, Opolka U, et al. (eds.). Neuro-Enhancement. Ethik vor neuen Herausforderungen. Paderborn: Mentis; 2009.

3. Schöne-Seifert B, Talbot D, (eds.). Enhancement. Die Ethische Debatte. Paderborn: Mentis; 2009.

4. Solomon L, Noll R, Mordkoff D. Cognitive Enhancement in Human Beings. Gender Medicine 2009; 6(2): 338-344.

5. Academy of Medical Sciences. Brain science, addiction and drugs. An Academy of Medical Sciences working group report chaired by Professor Sir Gabriel Horn FRS FRCP. http://www.acmedsci.ac.uk/viewFile/524414fc8746a.pdf. Accessed 26 October 2014. 
6. Repantis D, Schlattmann P, Laisney O, et al. Modafinil and methylphenidate for neuroanhancement in healthy individuals: A systematic review. Pharmacological Research 2010; 62: 187-206.

7. Ragan I, Bard I, Singh I. What should we do about student use of cognitive enhancers? An analysis of current evidence. Neuropharmacology 2013; 64: 588-595.

8. Barros D, Ortega F. Metilfenidato e Aprimoramento Cognitivo Farmacológico: representaçóes sociais de universitários. Saude Soc. São Paulo 2011; 20(2): 350-362.

9. Sepúlveda M, Roa J, Muñoz M. Estudio cuantitativo del consumo de drogas y factores sociodemográficos asociados en estudiantes de una universidad tradicional chilena. Revista Médica de Chile 2011; 139: 856-863.

10. Rodríguez J, Hernández E, Fernández AM. Descripción del consumo de drogas lícitas e ilícitas por género a través de la metodología de pares. Revista Médica de Chile 2007; 135: 449-459.

11. Finger G, Rodrigues da Silva E, Falavigna A. Use of methylphenidate among medical students: a systematic review. Revista da Associação Médica Brasileira 2013; 59(3): 285-289.

12. Mazzoglio y Nabar MJ, Algieri R, Dogliotti C, et al. Utilización de sustancias psicoactivas en alumnos de anatomía y su implicación en el aprendizaje. Educ Med 2011; 14(2): 129-132.

13. Juengst E. 1998. What does enhancement mean? In Parens E, (ed.). Enhancing human traits: Ethical and social implications. Washington: Georgetown University Press; 1998: 29-47.

14. Huaquín V, Loaíza R. Exigencias académicas y estrés en las carreras de la facultad de medicina de la universidad austral de chile. Estudios Pedagógicos 2004; 30: 39-59.

15. Acevedo M, Arango L, Blandón L, et al. Consumo de anfetaminas, para mejorar rendimiento académico, en estudiantes de la universidad de Manizales, 2008. Archivos de Medicina (Manizales) 2009; 9(1): 43-57.

16. CONACE (Consejo Nacional para el Control de Estupefacientes). 2009. Boletín Biblioteca Virtual, Available at http:// www.bibliodrogas.cl/bibliodrogas/boletines/BOLETIN\%20ENERO\%202009.pdf. Accessed 6 November 2014.

17. Duffau G. Consumo de elementos energéticos por estudiante de pre y postítulo. Revista Pediatría Electrónica 2010; 10(1): 2-3.

18. Campos P, Gómez A, Henríquez P. Percepción de los estudiantes de las carreras de Enfermería y Medicina de la Universidad Austral de Chile en relación al rendimiento académico asociado al uso de Modafinilo, durante el primer semestre del año 2012. Degree Thesis; 2012: http://cybertesis.uach.cl/tesis/uach/2012/fmc198p/doc/fmc198p.pdf. Accessed 13 January 2015.

19. Alarcón N, Obando G, Vivallo A, et al. Consumo de Psicotrópicos en la Facultad de Medicina de la Universidad Austral de Chile. Revista ANACEM 2010; 4(1): 23-31.

20. Dworkin R. Equality, luck, and hierarchy. Philosophy and Public Affairs 2003; 31(2): 190-198.

21. Cohen G. On the currency of egalitarian justice. Ethics 1989; 99(4): 906-944.

22. Arneson R. Equality and equal opportunity of welfare. Philosophical Studies 1989; 56: 77-93.

23. Cohen G. If You're an Egalitarian, How Come You're So Rich? Cambridge: Harvard; 2000.

24. Scheffler S. What is egalitarianism? Philosophy and Public Affairs 2003; 31(1): 5-39.

25. Anderson E. What is the point of equality. Ethics 1999; 109(2): 287-337.

26. Miller D. 1998. Equality and justice. In Mason E, ed. Ideals of equality. Oxford: Blackwell; 1998: 230-244.

27. Rawls J. Justice as Fairness. Cambridge, M.A.: Harvard University Press; 2001.

28. Rawls J. A Theory of Justice, Cambridge. M.A.: Harvard University Press; 1971.

29. Rawls J. Collected Papers. Cambridge, M.A.: Harvard University Press; 1999.

30. Arneson R. Against Rawlsian Equality of Opportunity. Philosophical Studies 1999; 93: 77-112.

31. Buchanan A. Equal Opportunity and Genetic Intervention. Social Philosophy and Policy Foundation 1995; 12(2): 105135.

32. Buchanan A, Brock D, Daniels N, et al. From Chance to Choice. Genetics and Justice. Cambridge: Cambridge University Press; 2000.

33. Boorse Ch. On the distinction between disease and illness. Philosophy and Public Affairs 1975; 5(1): $49-68$.

34. Salkever D. Updated estimates of earning benefits from reduced exposure of children to environmental lead. Environmental Research 1995; 70(1): 1-6.

35. Gottfredson L. Why G matters: the complexity of everyday life. Intelligence 1997; 24(1): 79-132.

36. Gottfredson L. Life, Death, and Intelligence. Journal of Cognitive Education and Psychology 2004; 4(1): $23-46$.

37. Whalley L, Deary I. Longitudinal cohort study of childhood IQ and survival up to age 76. British Medical Journal 2001; 
Justicia igualitaria y políticas de acceso al mejoramiento cognitivo farmacológico - Daniel Loewe 322: 819-822.

38. Holtug N. Equality and the Treatment-Enhancement distinction. Bioethics 2011; 25(3): 137-144.

39. Reiman J. Being fair to future people: the non-identity problem in the original position. Philosophical and public affairs 2007; 35(1): 69-92.

40. Dresler M, Sandberg A, Ohla K, et al. Non-pharmacological cognitive enhancement. Neuropharmacology 2013; 64: 529-543.

41. Savulescu J. Justice, Fairness, and Enhancement. Annals of the New York Academy of Sciences 2006; 1093: 321-338.

42. Farah M. Emerging ethical issues in neuroscience. Nature neuroscience 2002; 5(11): 1123-1129.

43. Boorse Ch. What a theory of mental health should be. Journal for the Theory of Social Behaviour 1976; 6(1): 61-84.

44. Boorse Ch. Health as a theoretical concept. Philosophy of Science 1977; 44(4): 542-573.

45. Daniels N. Just health care. New York: Cambridge University Press; 1985.

46. Daniels N. Normal functioning and the treatment-enhancement distinction. Cambridge Quarterly of Healthcare Ethics 2000; 9: 309-322.

47. Bunzl M. Comment on "health as a theoretical concept". Philosophy of Science 1980; 47(1): 116-118.

48. Trnka J. The Ethics of Cognitive Enhancement: Is it wrong to take 'smart drugs'?; 2009. Available at http://www.academia.edu/2914861/The_Ethics_of_Cognitive_Enhancement. Accessed 1 December 2014.

49. Reglamento de Ley No. 20.000 que sanciona el tráfico ilícito de estupefacientes y sustancias sicotrópicas y sustituye la ley No 19.366. Available at http://transparencia.redsalud.gov.cl/transparencia/public/sschiloe/archivos/942A99B63649DC78 E04001011F010649. Accessed 10 september 2014.

50. Farah M, Illes J, Cook-Deegan R, et al. Neurocognitive enhancement: what can we do and what should we do? Nature Reviews/ Neuroscience 2004; 5: 421-425.

51. Brant L, Carvalho T. Metilfenidato: medicamento gadget da contemporaneidade. Interface, Comunicação Saúde Educação 2012; 16(42): 623-636.

52. Dubljević V. Toward a Legitimate Public Policy on Cognitive Enhancement Drugs. AJOB Neuroscience 2012; 3(3): 2933.

53. Dubljević V. Prohibition or Coffee Shops: Regulation of Amphetamine and Methylphenidate for Enhancement Use by Healthy Adults. The American Journal of Bioethics 2013; 13(7): 23-33.

54. Gesang B. Perfektionierung des Menschen. New York, Berlin: Walter de Gruyter; 2007.

55. Bostrom N, Sandberg A. Cognitive Enhancement: Methods, Ethics, Regulatory Challenges. Sci Eng Ethics 2009; 15: 311-341.

56. Randall D, Shneerson J, File S. Cognitive effects of modafinil in students volunteers may depend on IQ. Pharmachology Biochemistry and Behavior 2005; 82(1): 133-139.

57. Glannon W. Psychopharmacological enhancement. Neuroethics 2008; 1: 45-54.

58. De Jongh R, Bolt I, Schermer M, et al. 2008. Botox for the brain: enhancement of cognition, mood and pre-social behavior and blunting of unwanted memories. Neuroscience and Biobehavioral Reviews 2008; 32: 760-776.

59. Bostrom N. Human Genetic Enhancement: A transhumanistic perspective. Journal of Value Inquiry 2003; 37(4): 493506.

60. Loewe D. Cognitive enhancement and the leveling of the playing-field. In: Jotterand F, Dubljevic V, (eds.). Cognitive Enhancement: Ethical and Policy Implications in International Perspectives. Oxford University Press; 2016: 219-236.

Recibido: 12 de agosto de 2015

Aceptado: 24 de noviembre de 2015 